\title{
Efectos de una Intervención Cognitivo-Conductual en el Aumento de Conductas de Autocuidado y Disminución del Estrés Traumático Secundario en Psicólogos Clínicos
}

\section{Effects of a Cognitive-Behavioral Treatment on the Increase of Self-Care Behaviors and Decrease of Secondary Traumatic Stress on Clinical Psychologists}

\author{
Cristóbal Guerra Vio* \\ Andrea Fuenzalida Vivanco \\ Carla Hernández Morales \\ Universidad del Mar, Chile
}

(Rec: 30 de Septiembre 2008 Acep: 10 de abril 2009)

\begin{abstract}
Resumen
En la presente investigación se evalúa la efectividad de un taller de autocuidado de orientación cognitivo conductual diseñado para ser aplicado a psicólogos clínicos con altos niveles de estrés traumático secundario. El objetivo del taller es aumentar la frecuencia de emisión de conductas de autocuidado y disminuir los niveles de estrés traumático secundario en los psicólogos. Mediante un diseño cuasi-experimental se evalúan los efectos del taller. Los resultados indican que, después de las 5 sesiones que dura el taller, los psicólogos que participaron en él $(\mathrm{n}=9)$ aumentaron significativamente sus conductas de autocuidado y disminuyeron significativamente sus niveles de desgaste. En cambio, los psicólogos que no participaron del taller $(\mathrm{n}=12)$ no variaron en la frecuencia del autocuidado y aumentaron el estrés traumático secundario. En virtud de estos resultados se concluye que el taller es efectivo y se constituye como una alternativa para prevenir y tratar el desgaste laboral en psicólogos clínicos.
\end{abstract}

Palabras clave: Taller de autocuidado, conductas de autocuidado, estrés traumático secundario, psicólogos clínicos.

\begin{abstract}
In the present investigation we evaluate the effectiveness of a group therapy program for self-care with cognitive-behavioral orientation, designed to be used on clinical psychologists with high levels of secondary traumatic stress. The object of this group therapy program is to increase the frequency of emissions of self-care behaviors and decrease the levels of secondary traumatic stress in psychologists. By a quasi-experimental design we evaluate the group therapy program effects. The results indicate that, after 5 sessions that lasts the group therapy program, the psychologists that participated in it $(n=9)$ increased significantly their self-care behaviors and decreased significantly their secondary traumatic stress levels. Instead, the psychologists that didn't participate in the group therapy program $(n=12)$ didn't show variation on self-care frequency and they increased secondary traumatic stress. These results are conclusive that the group therapy program is effective and it represents an alternative to prevent and treat work related burnout in clinical psychologists.

Key Words: Group therapy program of self-care, self-care behaviors, secondary traumatic stress, clinical
\end{abstract} psychologists. 


\section{Introducción}

En los últimos años ha existido un creciente interés por investigar el desgaste laboral en psicólogos y otros profesionales de ayuda, lo que se ha visto reflejado en el aumento de publicaciones referidas a este tema (Figley, 2002; Salston \& Figley 2003; Stamm, 2002; Valent, 2002). Este interés por estudiar los efectos adversos del trabajo de ayuda ha derivado en el estudio del autocuidado como variable asociada con la prevención y tratamiento del desgaste laboral (Morales, Pérez \& Menares, 2003; Guerra \& Lira, 2007; Radey \& Figley, 2007).

No obstante lo anterior, el autocuidado sigue siendo un constructo teórico ambiguo ya que se carece de investigaciones que permitan operacionalizarlo empíricamente. En la recopilación bibliográfica realizada sólo se hallaron referencias teóricas sobre el autocuidado (Arredondo, 2007; Hesse, 2002; Pearlman \& Saakvitne, 1995; Richardson, 2001; Saakvitne \& Pearlman, 1996), referencias de investigaciones descriptivas o correlaciónales (Betta, Morales, Rodríguez \& Guerra, 2007; Durruty, 2005; Hidalgo \& Moreira, 2000; Santana \& Farkas, 2007) o bien descripciones de talleres de autocuidado para profesionales (Claramunt, 1999; Gomez \& Gomez, 2006). No obstante, en ninguna de estas referencias se entregan datos cuantitativos referidos a los efectos de programas estructurados de autocuidado en población de psicólogos clínicos.

Es por esta razón que la presente investigación se propone, mediante un diseño cuasiexperimental, evaluar la eficacia de un taller cognitivo conductual, orientando al aumento de conductas de autocuidado y a la disminución de los niveles de estrés traumático secundario en psicólogos clínicos. Se hipotetiza que la intervención será efectiva al generar estos cambios en los psicólogos que participen del taller.

A continuación se ofrece una breve descripción de los fundamentos teóricos y empíricos que motivaron la presente investigación.

\section{Desgaste laboral y autocuidado}

Como ya se ha señalado, en la literatura existe abundante evidencia del desgaste laboral asociado a las profesiones de ayuda y a la práctica de la psicoterapia. Dentro de las posibles consecuencias adversas de este tipo de trabajo destacan constructos como el síndrome de burnout (Maslash, Schaufeli \& Leiter, 2001), la presencia de sintomatología ansiosa (Cazabat, 2002; Gentry, 2003) y depresiva (Barrera \& Guerra, 2007; Betta, et al, 2007) en los profesionales, el aumento de licencias medicas (Morales, Perez \& Menares, 2003), rotación de personal (Hidalgo \& Moreira, 2000), el aumento en el consumo de alcohol (Beaton \& Murphy, 1995) y el estrés traumático secundario (Figley, 2002; Salston \& Figley, 2003; Stamm, 2002; Valent, 2002).
Para esta investigación se optó por incluir al último de estos constructos: el estrés traumático secundario (en adelante ETS). El ETS es una forma de nominar los efectos negativos que se producen en las personas que trabajan en contacto directo con supervivientes de eventos traumáticos (Bride, Robinson, Yegidis \& Figley, 2004). Los autores indican que los efectos de la exposición secundaria a eventos traumáticos son equivalentes a los efectos de la exposición directa, ya que incluyen la misma sintomatología correspondiente al Trastorno por estrés postraumático, con la única diferencia que quien padece esta sintomatología es el profesional que ha atendido a la víctima directa.

Para prevenir y tratar éste y otros tipos de desgaste laboral, paulatinamente está cobrando relevancia el concepto de autocuidado, que corresponde a un constructo amplio que se define como el conjunto de acciones intencionadas que realiza una persona cualquiera para controlar los factores internos y externos, que pueden comprometer su vida y su desarrollo posterior (Uribe, 1999). Aplicado a la labor del psicólogo clínico se considera que el autocuidado está referido a las competencias del terapeuta para promover su salud y su calidad de vida de manera de detectar, enfrentar y resolver situaciones que generen desgaste laboral (Yáñez, 2005, en Durruty, 2005).

Para efectos de este artículo se utiliza la noción conductual del autocuidado, donde se lo considera como un conjunto amplio de conductas que, al ser emitidas, se asocian a bajos niveles de desgaste laboral (Guerra, Rodríguez, Morales \& Betta, en prensa).

Bajo esta concepción del autocuidado, Fuenzalida, Hernández y Guerra (2008) diseñan un programa de intervención grupal para desarrollar conductas de autocuidado y de ese modo disminuir los niveles de ETS en psicólogos clínicos. Para el diseño de este programa de intervención se consideraron 4 referencias; 1 . El planteamiento de Báguena (2001) sobre la efectividad de las técnicas de intervención cognitivo conductuales para el tratamiento del estrés postraumático; 2. Los antecedentes empíricos que indican la existencia de relaciones inversas entre una serie de conductas de autocuidado y el ETS (Betta, et al 2007); 3. La propuesta de intervención cognitivo conductual para prevenir y tratar el síndrome de burnout de Gómez y Gómez (2006); y 4. El programa de tratamiento para el ETS desarrollado por Gentry, Baranowsky y Dunning (2002).

El objetivo principal del taller cognitivo conductual de autocuidado (Fuenzalida et al, 2008) es aumentar la frecuencia de emisión de conductas de autocuidado y disminuir los niveles de ETS en psicólogos clínicos.

Para ello el taller se estructura en 5 sesiones de la siguiente manera:

Sesión 1. Psicoeducación sobre ETS y autocuidado, e identificación de las fuentes de estrés laboral;

Sesión 2. Capacitación en la distribución del tiempo en el trabajo y en técnicas de relajación; 
Sesión 3. Capacitación en comunicación asertiva en el trabajo;

Sesión 4. Planificación de la ejecución de conductas de autocuidado fuera del trabajo; y Sesión 5. Evaluación de los cambios producidos en el taller y planificación de la generalización de los resultados.

\section{Método}

\section{Participantes.}

Para conformar la muestra final de la investigación se solicitó a 43 psicólogos clínicos de la $\mathrm{V}$ región del país que respondieran la batería de instrumentos para seleccionar a los que presentaban ETS. 21 de estos psicólogos presentaban niveles de ETS por sobre el puntaje de corte de la escala utilizada para medir este constructo (38 puntos; Bride, 2007).

De este modo, se conformaron dos grupos: un grupo control compuesto por 12 psicólogos y un grupo experimental compuesto por 9 profesionales. La Tabla 1 resume las características de ambos grupos.

\section{Instrumentos}

Se utilizaron los siguientes instrumentos:

1. Cuestionario de variables sociodemográficas: Cuestionario de autorregistro destinado a obtener datos descriptivos de la muestra: edad, género, estado civil, número de hijos, años de experiencia como psicólogo y años de experiencia como psicólogo clínico.

2. Escala de Conductas de Autocuidado para Psicólogos Clínicos (EAP; Guerra et al, en prensa): Consiste en una escala de autorregistro dirigida a cuantificar la frecuencia con que los psicólogos clínicos emiten conductas de autocuidado. Está conformada por 10 ítems, cada uno de los cuales representa una conducta de autocuidado específica. La escala de frecuencia de las puntuaciones va desde 0 (nunca) a 4 (muy frecuentemente). El puntaje total se calcula sumando las respuestas dadas por los psicólogos a los 10 ítems de la escala. Los puntajes finales pueden variar desde

Tabla 1. Descripción de la Muestra

\begin{tabular}{|c|c|c|}
\hline & $\begin{array}{l}\text { Grupo } \\
\text { Experimental }\end{array}$ & $\begin{array}{l}\text { Grupo } \\
\text { Control }\end{array}$ \\
\hline \multicolumn{3}{|l|}{ Edad (años) } \\
\hline Rango & $23-43$ & $32-47$ \\
\hline Media y desviación típica & $30,22(6,36)$ & $37,17(5,47)$ \\
\hline \multicolumn{3}{|l|}{ Género (n y $(\%))$} \\
\hline Femenino & $9(100 \%)$ & $6(50 \%)$ \\
\hline Masculino & $0(0 \%)$ & $6(50 \%)$ \\
\hline \multicolumn{3}{|l|}{ Estado Civil (n y (\%)) } \\
\hline Soltero & (3) $33,33 \%$ & (5) $41,66 \%$ \\
\hline Casado/conviviente & (6) $66,66 \%$ & (7) $58,33 \%$ \\
\hline \multicolumn{3}{|l|}{ Número de hijos } \\
\hline Rango & $0-3$ & $0-3$ \\
\hline Media y desviación típica & $0,78(1,09)$ & $1,58(1,08)$ \\
\hline \multicolumn{3}{|l|}{ Años como psicólogo } \\
\hline Rango & $1-16$ & $1-18$ \\
\hline Media y desviación típica & $5,11(5,39)$ & $8,92(4,52)$ \\
\hline \multicolumn{3}{|l|}{ Años como psicólogo clínico } \\
\hline Rango & $1-16$ & $1-15$ \\
\hline Media y desviación típica & $4,89(5,46)$ & $7,08(3,94)$ \\
\hline \multicolumn{3}{|c|}{ Frecuencia de autocuidado (EAP) } \\
\hline Rango & $19-33$ & $11-29$ \\
\hline Media y desviación típica & $18,5(5,5)$ & $21,8(5,4)$ \\
\hline \multicolumn{3}{|c|}{ Estrés Traumático Secundario (EETS) } \\
\hline Rango & $38-60$ & $38-61$ \\
\hline Media y desviación típica & $47,8(9,4)$ & $44,8(8,9)$ \\
\hline
\end{tabular}


Tabla 2. Diferencias en las variables descriptivas de la muestra en grupo experimental y grupo control

\begin{tabular}{llc}
\hline Edad (años) & $\mathrm{U}=20.00$ & $\mathrm{p}=0,02$ \\
Género & $\chi 2=6,30$ & $\mathrm{p}=0,01$ \\
Estado Civil & $\chi 2=0,15$ & $\mathrm{p}=0,69$ \\
Número de hijos & $\mathrm{U}=31.00$ & $\mathrm{p}=0,09$ \\
Años como psicólogo & $\mathrm{U}=26.00$ & $\mathrm{p}=0,05$ \\
Años como psicólogo clínico & $\mathrm{U}=31,50$ & $\mathrm{p}=0,10$ \\
\hline
\end{tabular}

0 a 40 puntos, a mayor puntaje obtenido mayor es la frecuencia de emisión de conductas de autocuidado. Puntajes entre 0 y 7 puntos indican que el psicólogo "nunca" practica el autocuidado, entre 8 y 15 se considera que lo practica "casi nunca", entre 16 y 23 puntos indica que lo practica "ocasionalmente", entre 24 y 31 puntos se considera que la persona "frecuentemente" utiliza las estrategias, y puntajes totales sobre 32 puntos indican que el psicólogo utiliza el autocuidado "muy frecuentemente".

La confiabilidad de la escala, en esta intervención, arrojo puntajes alfa de cronbach superiores a 0,71 en los distintos análisis realizados (tanto con la muestra de 43 psicólogos, como en las mediciones pre y post realizadas con los 21 psicólogos de los grupos control y experimental).

3. Escala de estrés traumático secundario (EETS; Bride, et al, 2004, adaptada a Chile por Guerra \& Saiz, 2007): Corresponde a una escala de autorregistro destinada a evaluar los síntomas de ETS en profesionales de ayuda. Está conformada por 17 ítems que miden síntomas de intrusión, evitación y activación asociados a la exposición indirecta a eventos traumáticos. Cada ítem tiene un rango de puntuación que indica la frecuencia de experimentación de los síntomas durante los últimos siete días. En esta escala de frecuencia las puntuaciones van desde 1 (nunca) a 5 (con mucha frecuencia). El puntaje total se obtiene sumando las respuestas de los 17 ítems. Bride (2007) indica que desde los 38 puntos el profesional presenta ETS. Además, el autor indica que bajo 28 puntos hay una sintomatología "muy baja"; entre 28 a 37 puntos la sintomatología es "baja", entre 38 y 43 puntos la sintomatología es "moderada"; entre 44 y 48 puntos la sintomatología es "alta" y sobre los 49 puntos la sintomatología es "severa".

La fiabilidad de la escala, en esta intervención, arrojo valores alfa de Cronbach superiores a 0,86 en los diversos análisis realizados (tanto con la muestra de 43 encuestados, como en las mediciones pre y post realizadas con los 21 psicólogos que finalmente participaron del estudio).

\section{Procedimiento.}

En un primer momento se aplicó la batería de instrumentos a 43 psicólogos clínicos (aplicación pretest). De ellos se seleccionó a los 21 profesionales que presentaban ETS y se conformaron los grupos control y experimental. El único criterio para distribuir a los participantes en los grupos fue la disponibilidad personal y de tiempo para participar del taller de 5 sesiones.

Nueve psicólogos manifestaron disponibilidad para participar del taller, mientras que los 12 restantes no lo hicieron $^{* *}$. De esta forma, el grupo experimental quedó conformado por 9 psicólogos y el grupo control por los 12 restantes.

El grupo experimental participó de 5 sesiones de 90 minutos, con una frecuencia semanal. Estas sesiones fueron realizadas por dos psicólogas quienes siguieron la estructura del Taller cognitivo conductual de autocuidado para psicólogos clínicos de Fuenzalida, et al (2008). Es necesario destacar que en todas las sesiones se incluyó la aplicación de la batería de instrumentos y la realización de gráficos que expresan los cambios en el ETS y autocuidado evidenciados por los participantes durante las 5 sesiones.

Finalmente se aplicó la medición post intervención tanto al grupo experimental como al grupo control.

\section{Resultados}

Con el objeto de evaluar la equivalencia entre el grupo experimental y el grupo control se procedió a realizar una serie de contrastes relacionados tanto con las variables descriptivas de la muestra como con las variables objeto de la intervención.

Como puede observarse en la tabla 2, los contrastes realizados con $\chi^{2}$ y $\mathrm{U}$ de Mann-Whitney indican que el grupo experimental y control no presentan diferencias estadísticamente significativas en las variables estado civil, número de hijos y años como psicólogo clínico. No obstante, sí se observaron diferencias estadísticamente significativas en las variables edad, años como psicólogo y género. Específicamente se aprecia que la edad y la cantidad de años como psicólogo clínico de los profesionales del grupo experimental es significativamente menor que la de los psicólogos del grupo control. Además existe una clara diferencia en cuanto al género, ya que en el grupo experimental sólo participan psicólogos de género femenino, mientras que en el grupo control hay una participación de la misma cantidad de hombres que de mujeres. Las

\footnotetext{
* Hay que destacar que la principal razón que dieron estos 12 psicólogos para no participar del grupo experimental fue la falta de tiempo para asistir al taller.
} 
implicancias de estas diferencias serán abordadas en la sección discusión

En cuanto a la equivalencia entre el grupo experimental y el grupo control en las variables objeto de la intervención se aprecia que no existe diferencia estadísticamente significativa en la medición pretest. Es decir, ambos grupos son equivalentes en la frecuencia de emisión de conductas de autocuidado y en los niveles de ETS, según los análisis realizados con U de Mann-Whitney (ver Tabla 3).

Tabla 3. Diferencias en las variables objeto de intervención, en el pre test, entre el grupo experimental y el grupo control

\begin{tabular}{lll}
\hline EAP & $\mathrm{U}=30,00$ & $\mathrm{p}=0,08$ \\
EETS & $\mathrm{U}=46.00$ & $\mathrm{p}=0,56$ \\
\hline
\end{tabular}

Esta equivalencia en la frecuencia de emisión de conductas de autocuidado puede profundizarse en la figura 1, ya que ambos grupos se ubican en la categoría de práctica “ocasional” del autocuidado según los criterios para interpretar los puntajes en esa escala (Guerra et al, en prensa). Algo similar ocurre en el caso de los niveles de ETS, ya que tanto el grupo experimental como el grupo control poseen un puntaje promedio en la EETS que los ubica en la categoría de "alta" sintomatología de ETS según las normas de Bride (2007) (ver Figura 2).

Los datos referidos al aumento de conductas de autocuidado (ver gráfico 1) indican que, en la evaluación pretest, ambos grupos se ubican en la categoría de práctica "ocasional" del autocuidado. Específicamente el grupo experimental puntúa en promedio $18,5(\mathrm{DE}=5,5)$ y el grupo control 21,8 (DE=5,3). En la evaluación postest el grupo experimental presenta una media de $23,5(\mathrm{DE}=4,3)$, lo cual califica en el rango práctica "frecuente" de autocuidado. En relación al grupo control en la evaluación postest, éste presenta una media de 21,5 ( $\mathrm{DE}=5,4)$, lo cual indica que se mantiene en el rango de práctica "ocasional" de conductas de autocuidado.

Con respecto a los resultados de la intervención en el ETS, en el gráfico 2 puede apreciarse la diferencia en

Figura 1. Puntajes promedio de autocuidado Pre y Post en ambos grupos, según EAP

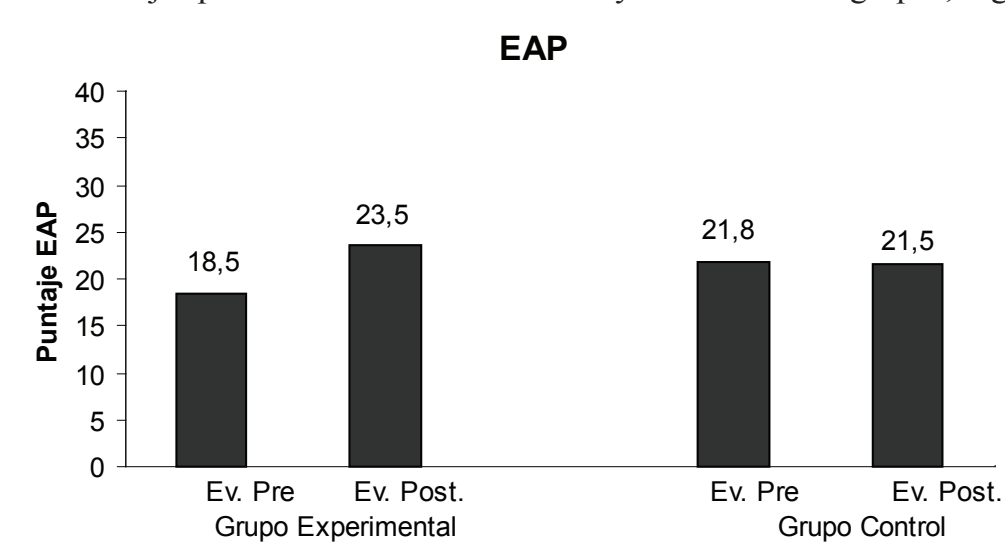

Figura 2. Puntajes promedio de ETS Pre y Post en ambos grupos, según EETS

\section{EETS}

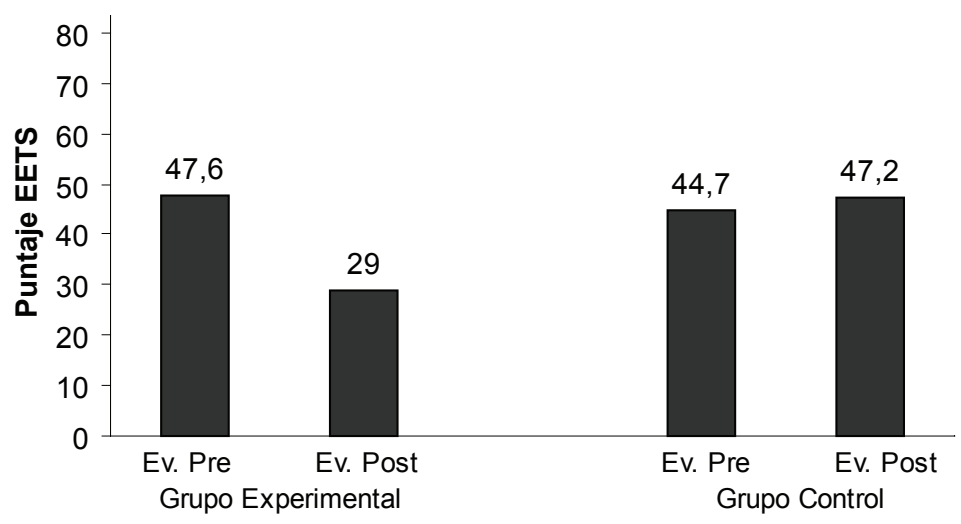


los puntajes promedio que obtuvo el grupo experimental y el grupo control en las mediciones pretest y postest en el ETS. En la evaluación pretest, el grupo experimental presenta una media de $47,6(\mathrm{DE}=9,4)$ y el grupo control muestra una media de 44,7 (DE= 8,9) lo cual corresponde, en ambos casos, al rango de "altos" niveles de ETS. Luego de la aplicación del taller, en la evaluación post, el grupo experimental presenta una media de 29,0 $(\mathrm{DE}=7,0)$ puntos ubicándose en un rango de puntajes "bajos" en los niveles de ETS según Bride (2007). Por su parte, el grupo control en la evaluación post presenta una media de 47,2 (DE= $8,9)$, lo cual indica que se mantiene en el rango de "altos" niveles de ETS.

Para evaluar si estas diferencias son estadísticamente significativas se realizaron contrastes con la prueba $\mathrm{z}$ de Wilcoxon. En el caso del grupo experimental, estos análisis confirman las hipótesis planteadas inicialmente, ya que indican que las diferencias son estadísticamente significativas en ambos casos, es decir, el grupo experimental aumentó la frecuencia con que emiten conductas de autocuidado y disminuyó los niveles de ETS respecto a la medición inicial (ver Tabla 4).

Tabla 4. Diferencias en el grupo experimental, entre el pretest y el postest, en las variables objeto de intervención

\begin{tabular}{lll}
\hline EAP & $\mathrm{z}=-2,55$ & $\mathrm{p}=0,01$ \\
EETS & $\mathrm{z}=-2,67$ & $\mathrm{p}=0,00$ \\
\hline
\end{tabular}

Por su parte, los análisis estadísticos indican que, tal como se esperaba, el grupo control no presentó diferencias estadísticamente significativas en la frecuencia de emisión de conductas de autocuidado entre las mediciones pretest y postest. No obstante, se aprecia que sí hay diferencia estadísticamente significativa en los niveles de desgaste, existiendo un aumento en los niveles de ETS (ver tabla 5).

Tabla 5. Diferencias en el grupo control, entre el pretest y el postest, en las variables objeto de intervención

\begin{tabular}{lll}
\hline EAP & $\mathrm{z}=-0,73$ & $\mathrm{p}=0,46$ \\
EETS & $\mathrm{z}=-2,04$ & $\mathrm{p}=0,04$ \\
\hline
\end{tabular}

Por otro lado, la Tabla 6 muestra la comparación entre las variables objeto de intervención en el postest entre el grupo experimental y control, según la prueba $U$ de Mann-Whitney. En esta tabla se aprecia que, aún cuando el grupo experimental aumentó la frecuencia de conductas de autocuidado, no se evidencian diferencias estadísticamente significativas con respecto al grupo control. Las implicancias de este resultado inesperado se abordan en la sección discusión.

Respecto al ETS se observa que sí existe diferencia estadísticamente significativa en los niveles de ETS entre el grupo experimental y control en la medición postest. Es decir el grupo control, que no recibió la intervención, presenta mayores niveles de ETS que el grupo experimental, que sí recibió la intervención.

Tabla 6. Diferencias en las variables objeto de intervención, en el post test, entre el grupo experimental y el grupo control

\begin{tabular}{lll} 
EAP & $\mathrm{U}=43,00$ & $\mathrm{p}=0,43$ \\
EETS & $\mathrm{U}=2,50$ & $\mathrm{p}=0,00$ \\
\hline
\end{tabular}

Finalmente, en la figura 3 se presenta el detalle de los puntajes de la EAP y EETS que obtuvieron los 9 participantes del grupo experimental en cada una de las 5 sesiones. Hay que destacar que la sesión 1 corresponde a la evaluación pretest y la sesión 5 corresponde a la evaluación postest.

En esta figura se puede apreciar que el taller resultó en el aumento de los niveles de autocuidado en 8 de los 9 psicólogos que participaron del taller $(88,8 \%)$, ya que hay un participante que disminuyó levemente los puntajes en la EAP respecto de la medición pretest (de 22 puntos a 21 puntos).

Respecto al ETS, en esta figura se aprecia que el taller resultó en la disminución de este tipo de sintomatología en el $100 \%$ de los psicólogos que participaron del grupo experimental, no obstante en uno de los casos esta disminución en los niveles de ETS fue parcial (bajando de 59 a 39 puntos), ya que se mantuvo por sobre el puntaje de corte de 38 puntos.

\section{Discusión}

En la presente investigación participaron 21 psicólogos que presentaban indicadores de ETS. Estos psicólogos fueron divididos en dos grupos equivalentes en el nivel de ETS que presentaban sus integrantes. Además, ambos grupos presentaban un nivel similar de ejecución de prácticas de autocuidado.

El grupo experimental estuvo conformado por 9 psicólogos, a quienes se les aplicó el taller cognitivo conductual dirigido a aumentar la frecuencia de emisión de conductas de autocuidado y a disminuir sus niveles de ETS. Los 12 psicólogos restantes conformaron el grupo control que no participó del taller.

Los resultados indican que los psicólogos del grupo experimental aumentaron significativamente la frecuencia de emisión de prácticas de autocuidado y disminuyeron significativamente sus niveles de ETS después de haber participado del taller. Por su parte, los integrantes del grupo control, que no participaron del taller, se mantuvieron estables en la frecuencia de emisión de conductas de autocuidado y aumentaron significativamente sus niveles de ETS. 
Figura 3. Puntajes Individuales (EETS y EAP) del grupo experimental $(\mathbf{\square}=$ EETS. $\boldsymbol{\Delta}=$ EAP)
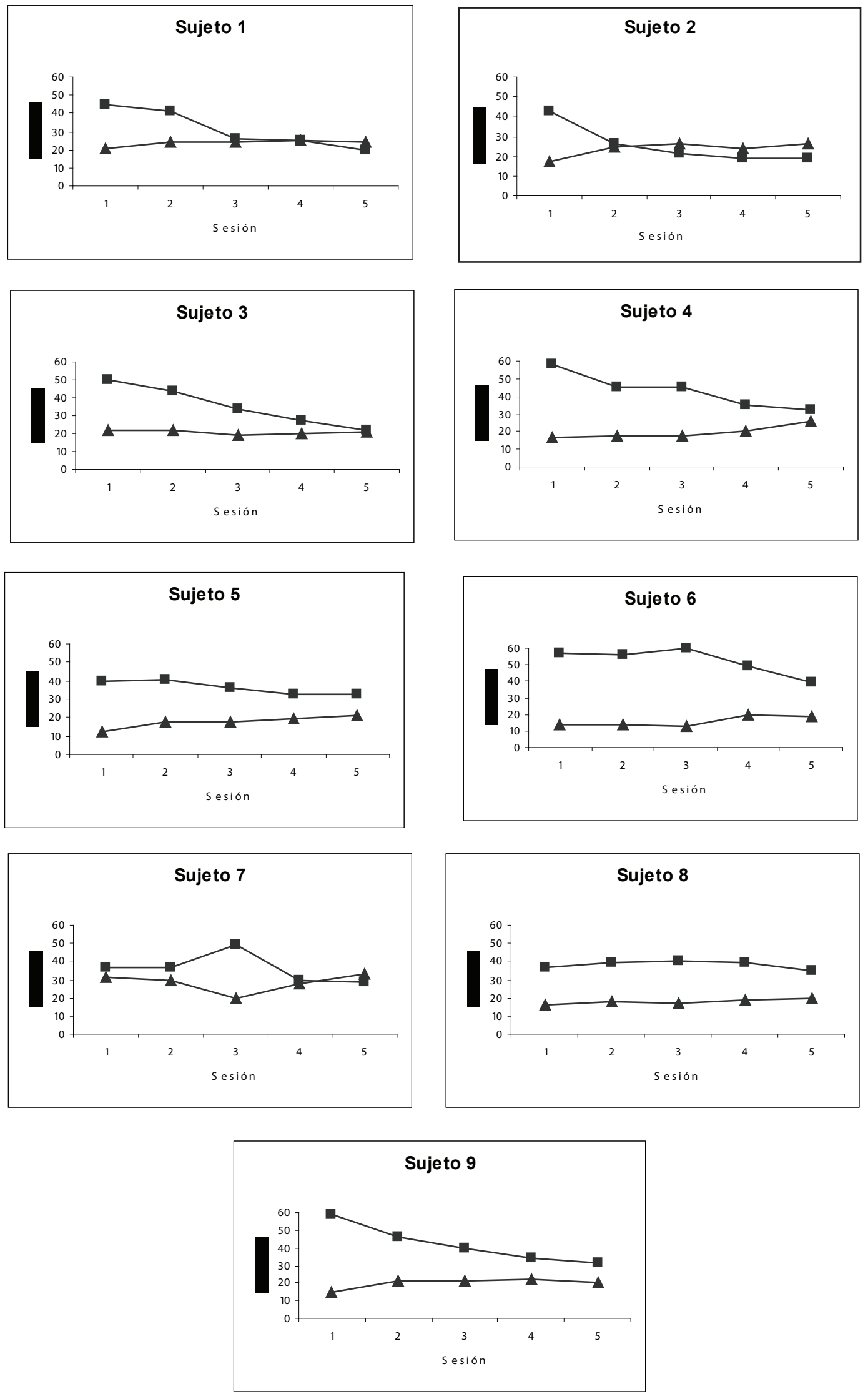
Pese a estos resultados, y a que en la medición postest el grupo experimental presenta mayores puntajes en autocuidado respecto al grupo control, la comparación postest referida a la variable autocuidado indica que estas diferencias entre ambos grupos no son estadísticamente significativas. Este resultado inesperado pudiera tener su origen en la forma de medición del autocuidado ya que, pese a utilizar un instrumento que ha demostrado adecuados índices de fiabilidad y validez en diferentes investigaciones (Guerra et al, en prensa; Guerra, Mujica, Nahmias y Rojas, 2008), la EAP corresponde a una escala que mide de forma acotada 10 conductas de autocuidado. Por lo tanto, pudiera ser que los psicólogos del grupo experimental hayan aumentado la frecuencia de ejecución de conductas de autocuidado no incluidas en la escala. Esta posibilidad es coherente con la visión conductual del autocuidado que lo considera como un conjunto amplio de estrategias prácticas que tienen como elemento común el disminuir el desgaste laboral (Guerra et al, en prensa).

Además, el análisis realizado en cada uno de los grupos confirma esta idea, ya que el grupo experimental, que aumentó significativamente la frecuencia del autocuidado, disminuyó significativamente los niveles de ETS. En cambio el grupo control, que se mantuvo estable en la emisión de conductas de autocuidado, aumentó los niveles de ETS. Por otro lado, el contraste estadístico realizado post test indica que el grupo experimental posee niveles de ETS significativamente más bajos que los del grupo control.

Estos resultados complementan lo señalado en investigaciones previas sobre la relación inversa entre el desgaste laboral y el autocuidado (Betta et al, 2007; Guerra et al, en prensa) y permiten avanzar en la formulación de relaciones de causalidad entre ambas variables. En este sentido, los resultados de la intervención apoyan la idea que el aumento en la frecuencia de emisión de conductas de autocuidado se constituye como la causa de la disminución de los niveles de desgaste laboral (en este caso de ETS).

De esta forma se aprueban las hipótesis del estudio, es decir, se considera que el taller cognitivo conductual implementado es efectivo, ya que potencia el aumento de la frecuencia de emisión de conductas de autocuidado en los participantes y la disminución de los niveles de ETS. Los análisis individuales realizados apoyan la efectividad de la intervención, debido a que en el $88 \%$ de los participantes se evidenció un aumento en los niveles de autocuidado medidos por la EAP y en el $100 \%$ de los participantes del taller se observó una disminución en los niveles de ETS.

Pese a lo alentador de estos resultados, hay que destacar que el estudio presenta a lo menos dos grandes limitaciones; primero, se trabajó con una muestra pequeña de profesionales, lo que impide la generalización de estos resultados a la población de psicólogos clínicos; segundo, el grupo experimental difiere del grupo control en el género, edad y años de experiencia laboral de sus integrantes, por lo que estas variables pudieran incidir en los resultados obtenidos.

Debido a lo anterior se considera relevante continuar realizando investigaciones en el tema del autocuidado que permitan operacionalizarlo. Específicamente se sugiere realizar estudios con muestras más amplias, además de utilizar criterios más precisos en la asignación de los participantes a los grupos de investigación. Por otro lado, pudiera ser interesante realizar estudios que incorporen muestras de diferentes profesiones y que consideren otras formas de desgaste laboral, como por ejemplo el síndrome de burnout.

Para finalizar se considera que esta investigación constituye un aporte en el estudio del autocuidado, ya que entrega antecedentes empíricos que permiten avanzar en su operacionalización. Además, los resultados de este estudio apoyan la idea de la importancia del autocuidado como forma de prevenir y tratar los efectos del desgaste laboral en psicólogos clínicos.

\section{Referencias}

Arredondo, V. (2007). Autocuidado en equipos psicosociales: El lugar de lo colectivo. En: V. Arredondo, \& E. Toro (Eds.). Violencia sexual infantil: debates, reflexiones y prácticas críticas, 90-97. Valparaíso, Chile: Editado por Paicabi-Sename.

Baguena, M. J. (2001). Tratamientos psicológicos eficaces para el estrés postraumático. Psicothema,13, 479-492.

Barrera, P. \& Guerra, C. (2007). Creencias de profesionales que atienden a Víctimas de Abuso Sexual Infantil respecto de la influencia del Proceso Legal en su Desgaste y Satisfacción Laboral. En: Corporación de Asistencia Judicial (Ed.). Atención a Victimas de Delitos Violentos: Reflexiones desde la Práctica, 177-192. Viña del Mar, Chile: Ril Editores.

Beaton, R. D. \& Murphy, S. A. (1995). Working with people in crisis: Research implications. En: Ch. Figley (Ed.). Compassion fatigue: Coping with secondary traumatic stress disorder in those who treat the traumatized, 51-81. New York: Brunner/Mazel Publishers.

Betta, R., Morales, G., Rodríguez, K. \& Guerra, C. (2007). La frecuencia de emisión de conductas de autocuidado y su relación con los niveles de estrés traumático secundario y depresión en psicólogos clínicos. Pensamiento psicológico, 3, 9-19.

Bride, B. E. (2007). Prevalence of Secondary Traumatic Stress among Social Workers. Social Work 52 (1), 63 - 70.

Bride, B. E., Robinson, M. M., Yegidis, B. \& Figley, Ch. (2004). Development and validation of the Secondary Traumatic Stress Scale. Research on Social Work Practice, 14(1), 27-35.

Cazabat, E. (2002). Desgaste por empatía. Extraído el 18 de mayo de 2007 desde http://www.interpsiquis.com/2002/areas/at8.

Claramunt, C. (1999). Ayudándonos a Ayudar a Otros. Guía para el Autocuidado de Quienes Trabajan en el Campo de la Violencia Intrafamiliar. San José, Costa Rica: Organización Panamericana de la Salud.

Durruty, P. (2005). Ejes de autocuidado para el terapeuta orientado a la prevención del síndrome de burnout desde la perspectiva de la asertividad generativa. Tesis de licenciatura no publicada, Universidad de Chile, Santiago, Chile.

Figley, Ch. R. (2002). Treating compassion fatigue. New York: BrunnerRoutledge.

Fuenzalida, A., Hernández, C. \& Guerra, C. (2008). Diseño de un programa de autocuidado cognitivo conductual para psicólogos clínicos. Mansucrito no publicado.

Gentry, J. E. (2003). Desgaste por empatía: El desafío de la transformación. Revista de Psicotrauma para Iberoamérica, 2, 4-17. 
Gentry, J. E., Baranowsky, A. B. \& Dunning, K. (2002). The Accelerated Recovery Program (ARP) for Compassion Fatigue. En: Ch. Figley (Ed.), Treating Compassion Fatigue, 123- 138. New York, London: Brunner- Routledge.

Gómez, I., y Gómez, X. (2006). Síndrome de Burnout, intervención preventiva: Manual para psicólogos. Cali: Sello Editorial Javeriano.

Guerra, C. \& Lira, G. (2007). Medición del desgaste profesional en una muestra de profesionales de la Corporación Paicabi: Una aproximación reflexiva al concepto de autocuidado en equipos que trabajan en maltrato infantil. En: V. Arredondo \& E. Toro (Eds.). Violencia sexual infantil: debates, reflexiones y prácticas críticas, 114 - 131. Valparaíso, Chile: Editado por Paicabi-Sename.

Guerra, C., Mujica, A., Nahmias, A., \& Rojas, N. (2008). Examen psicométrico de la Escala de conductas de autocuidado para psicólogos clínicos. Manuscrito no publicado.

Guerra, C., Rodríguez, K., Morales, G., \& Betta, R. (en prensa). Validación inicial de la Escala de conductas de autocuidado para psicólogos clínicos.

Guerra, C. \& Saiz, J. L. (2007). Examen psicométrico de la Escala de Estrés Traumático Secundario: Un estudio en profesionales chilenos. Psicología Conductual, 15(3), 441- 456.

Hesse, A. (2002). Secondary trauma: How working with trauma survivors affects therapists. Clinical Social Work Journal, 30, 293-309.

Hidalgo, N. \& Moreira, E. (2000). Autocuidado, Trastorno Psicológico y Rotación de Equipos de Salud Mental. Santiago: Departamento de Psicología, Universidad de Chile.

Maslach, C., Schaufeli, W. \& Leiter, M. (2001). Job Burnout. Annual Review of Psychology, 52, $397-422$.
Morales, G., Pérez, J. \& Menares, M. (2003). Procesos emocionales de cuidado y riesgo en profesionales que trabajan con el sufrimiento humano. Revista de Psicología de la Universidad de Chile, 12 9-25.

Pearlman, L., A. \& Saakvitne, K. W. (1995). Treating Therapist with Vicarious Traumatization and Secondary Traumatic Stress Disorders. En: Ch. Figley (Ed.). Compassion fatigue: Coping with secondary traumatic stress disorder in those who treat the traumatized, 150-177. Nueva York: Brunner/Mazel.

Radey, M. y Figley, C. R. (2007). The social psychology of compassion. Clinical Social Work Journal, 35, 207-214.

Richardson, J. I. (2001). Guidebook on vicarious trauma: Recommended solutions for anti-violence workers. Ottawa, ON: Health Canada.

Saakvitne, K. W. \& Pearlman, L. A. (1996).Transforming the Pain: A Workbook on Vicarious Traumatization for Helping Professionals Who Work with Traumatized Clients. New York: W. W. Norton \& Company Inc.

Salston, M. D. \& Figley, Ch. (2003). Secondary traumatic stress: Effects of working with survivors of criminal victimization. Journal of Traumatic Stress, 16, 167- 174.

Santana, I. \& Farkas, Ch. (2007). Estrategias de Autocuidado en Equipos Profesionales que Trabajan en Maltrato Infantil. Psykhe, 16, 77-89.

Stamm, B. H. (2002). Measuring Compassion Satisfaction as Well as fatigue: Developmental History of the Compassion Satisfaction and Fatigue Test. En: Ch. Figley (Ed.). Treating Compassion Fatigue, $107-$ 122. New York, London: Brunner- Routledge.

Uribe, T. (1999). El Autocuidado y su papel en la Promoción de la Salud. Revista Investigación y Educación en Enfermería, 17, 109-118.

Valent, P. (2002). Diagnosis and treatment of helper stresses, traumas, and illnesses. En: Ch. Figley (Ed.). Treating compassion fatigue, 17-37. New York Brunner-R. 